\section{Medical Device Risk Assessment Based on Ordered Weighted Averaging Aggregation Operator}

\author{
Fereshteh Azadi Parand ${ }^{1,2 * \odot}$, Ali Tavakoli-Golpaygani³, \\ Farzaneh Rezvani4
}

\begin{abstract}
Background: A medical device is any instrument, apparatus, implement, machine, appliance, software, material, which is intended material, to be utilized, either alone or in combination, for medical purpose. These devices should work precisely and the maintenance program of them has also a key role to achieve this goal. Many of the maintenance programs have not considered important functional parameters such as equipment type, risk factors, and expert opinion.
\end{abstract}

Objective: The purpose of this study is to present a novel fuzzy method for medical device risk assessment. The obtained values for risk could be used to prioritize maintenance operations by considering allocation budget.

Material and Methods: This experimental study aims to make a new application of Ordered-Weighted Average operator in aggregation of different parameters for calculating Risk Priority Number. This model is a fuzzy multi-criteria decision making approach based on risk maintenance framework for medical device prioritization.

Results: A limited budget is one of the barrier in medical centers. The suggested framework presents a simple and reliable method to choose the best maintenance strategy for each kind of medical device by considering budget limitation. Based on obtained results from numerical model, defibrillators and surgical suction have respectively the highest and the lowest priority in mentioned example.

Conclusion: Risk prioritization of medical devices is valuable because the medical centers can prioritize maintenance operations and thereby to establish preference of maintenance strategy. Implementation of our proposed maintenance program has many effective results in medical center budgets.

Citation: Azadi Parand F, Tavakoli-Golpaygani A, Rezvani F. Medical Device Risk Assessment Based on Ordered Weighted Averaging Aggregation Operator. J Biomed Phys Eng. 2021;11(5):621-628. doi: 10.31661/jbpe.v0i0.1133.

\section{Keywords}

Medical Device; Maintenance Program; Risk Priority; Fuzzy Logic; Ordered Weighted Averaging Operator; Risk Assessment

\section{Introduction}

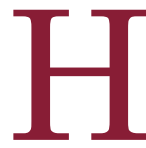

aving medical devices with correct performance and without any health hazards is critical for healthcare centers in the world. A medical device is any instrument, apparatus, implement, machine, appliance, implant, reagent for in vitro use, software, material or other similar or related article, intended to be utilized, either alone or in combination, for medical purposes to evaluate, recognize and treat diseases or prevent of them. These devices should work precisely according to reliability standards of healthcare services. Maintenance programs of medical devices are unacceptable and inefficient based on pe-
${ }^{1}$ PhD, Assistant Profes-

sor, Department of Math

and Computer Science,

Allameh Tabataba'i Uni-

versity, Tehran, Iran

${ }^{2} \mathrm{PhD}$, Visiting Fellow,

School of Computing,

Eng and Math, Western

Sydney University, Syd-

ney, Australia

${ }^{3} \mathrm{PhD}$, Assistant Profes-

sor, Department of

Biomedical Engineering,

Research Center of Tech-

nology and Engineer-

ing, Standard Research

Institiute, Karaj, Iran

${ }^{4} \mathrm{MSc}$, Department

of Math and Com-

puter Science, Allameh

Tabataba'i University,

Tehran, Iran

*Corresponding author: Fereshteh Azadi Parand Department of Math and Computer Science, Allameh Tabataba'i University, Tehran, Iran E-mail: parand@atu.ac.ir

Received: 30 January 2019 Accepted: 12 March 2019 
riodic visits, device age, professional liability and the main mission of medical centers and regardless of environmental conditions. The prioritization of medical devices was a fund project for health maintenance system (HMS) base on risk score of risk-based management programs. Clinical engineering departments of medical centers and hospitals have been developing maintenance programs such as medical equipment management program (MEMP) to reduce risks related to medical devices and increase their safety for patients and medical staff members. Calculating Risk Priority Number (RPN) is the first step in maintenance program for medical devices.

The Joint Commission on Accreditation of Healthcare Organizations (JCAHOs) have presented a series of medical equipment standards and have forced medical centers and hospitals to use their own risk management instruments to determine equipment implied in the MEMP in US [1]. Fennigkoh and Smith suggested a device classification plan based on three criteria, including maintenance requirements, physical harm/risk and equipment function [2]. Moreover, they calculated equipment management number and classified medical devices based on calculating scores to the three specified criteria. In 2004, JCAHO introduced the standard EC6.10 and confirmed the Fennigkoh and Smith procedure [3]. This procedure was used in many medical centers and hospitals widespread. Although this procedure, which is not dependent on parameters of risk factor, calculated an arithmetic average based on three factors, it is not appropriate for risk management. As Tawfik et al. has shown in their study, these shortcomings could investigate some critical equipment, such as steam sterilizers, hematology analyzers for low scores based on equipment function and physical harm to be considered as low risk [4].

\section{Material and Methods}

In this experimental study, American Society for Healthcare Engineering (ASHE) in 1996, presented a method to calculate the RPN of medical devices according to the five criteria as follows: clinical application (A), equipment function $(\mathrm{E})$, environmental use (U), preventive maintenance requirements $(\mathrm{P})$, probability of equipment failure (F), and a total score (T) which is calculated for each component using the equation (1) [5].

$$
\mathrm{T}=\mathrm{E}+\mathrm{A}+(\mathrm{P}+\mathrm{F}+\mathrm{U}) / 3
$$

Although all these methods consider some important factors and points, they have some drawbacks as follows:

1- All of the factors have the same weight but the relative importance has been overlooked.

2- Evaluations have not carried out between different experts.

3- Cost and profitability factors have not been mentioned.

In this study, a fuzzy logic approach for calculation RPN is introduced to overcome the traditional FMEA weakness due to investigation of uncertainties obtained from experts' different opinions, which relate linguistic variables to RPN factors. In addition, particular weights have been assigned not only to experts' different opinions but also to RPN factors.

Ordered Weighted Average Operator (OWA)

In 1988, Yager presented the ordered weighted averaging (OWA) operator [6]. In a classic definition, an OWA operator of dimension $n$ is a mapping as the equation (2), (3), (4).

$$
\mathrm{F}: R^{\mathrm{n}} \rightarrow R
$$

Which has an associated $\mathrm{n}$ vector $\mathrm{W}$ as follows:

$$
\begin{aligned}
& \mathrm{W}=\left[\mathrm{w}_{1}, \mathrm{w}_{2}, \ldots ., \mathrm{w}_{\mathrm{n}}\right]^{\mathrm{T}} \\
& \text { Such that } \mathbf{w}_{\mathrm{i}} \mathbf{\epsilon}[\mathbf{0 , 1}], \\
& \sum \mathrm{w}_{\mathrm{i}}=1 \mathrm{i}=1,2 \ldots \mathrm{n}
\end{aligned}
$$

Where $b_{j}$ is the $j$-th element of largest set of fused objects, $\mathrm{a}_{1}, \mathrm{a}_{2}, \ldots, \mathrm{a}_{\mathrm{n}}$. The value of $F\left(a_{1}, a_{2}\right.$, ,$\left.a_{\mathrm{n}}\right)$ can determine the aggregated value of objects $a_{1}, a_{2}, \quad, a_{\mathrm{n}}$. Particularly, a weight $w_{i}$ is 
Medical Device Risk Assessment

associated with an ordered position $i$ of the elements. One of the well-known characteristics of the OWA operators is including operators for the appropriate selection of the vector W, such as (5):

$1 . \mathrm{W}=[0,0, . ., 1]^{\mathrm{T}}, F\left(\mathrm{a}_{1}, \mathrm{a}_{2}, \ldots, \mathrm{a}_{\mathrm{n}}\right)=\operatorname{Min}\left(\mathrm{a}_{\mathrm{i}}\right)$

2. $\mathrm{W}=[1,0, \ldots, 0]^{\mathrm{T}}, \quad F\left(\mathrm{a}_{1}, \mathrm{a}_{2}, \ldots, \mathrm{a}_{\mathrm{n}}\right)=\operatorname{Max}\left(\mathrm{a}_{\mathrm{i}}\right)$

3. $\mathrm{W}=[1 / \mathrm{n}, 1 / \mathrm{n}, \ldots, 1 / \mathrm{n}]^{\mathrm{T}}, F\left(\mathrm{a}_{1}, \mathrm{a}_{2}, \ldots, \mathrm{a}_{\mathrm{n}}\right)=\operatorname{Mean}\left(\mathrm{a}_{\mathrm{i}}\right)$

Yager showed that OWA operators satisfied the commutativity, monotonicity and idem potency properties and were bound by the Max and Min operators,

$$
\operatorname{Min}\left(\mathrm{a}_{\mathrm{i}}\right) \leq F\left(\mathrm{a}_{1}, \mathrm{a}_{2}, \ldots, \mathrm{a}_{\mathrm{n}}\right) \leq \operatorname{Max}\left(\mathrm{a}_{\mathrm{i}}\right)
$$

Since this operator (OWA) was bounded by the Max (or) and the Min (and), Yager introduced the orness measure of the aggregation to characterize the type of aggregation performed for a particular value of the weighting vector, which is defined as (7):

$$
\text { Orness }(\mathrm{W})=\left(\sum(\mathrm{n}-\mathrm{i}) \times \mathrm{W}_{\mathrm{i}}\right) /(\mathrm{n}-1)
$$

The orness measure reflects the and-like or or-like aggregation result of an OWA operator, which is very important both in theory and applications.

$$
\begin{aligned}
& \text { Orness }\left([0,0, \ldots, 1]^{\mathrm{T}}\right)=0 \\
& \text { Orness }\left([1,0, \ldots, 0]^{\mathrm{T}}\right)=1 \\
& \text { Orness }\left([1 / \mathrm{n}, 1 / \mathrm{n}, \ldots, 1 / \mathrm{n}]^{\mathrm{T}}\right)=1 / 2
\end{aligned}
$$

Another measure introduced by Yager was the entropy associated with a weighting vector, used to calculate the amount of used information in arguments during an aggregation based on the vector W. This approach is based on the process of maximization of Dispersion, (11), has profit the most usage information theory.

$$
\operatorname{Disp}(\mathrm{W})=\sum \mathrm{W}_{\mathrm{i}} \ln \left(\mathrm{W}_{\mathrm{i}}\right) \mathrm{i}=1, . ., \mathrm{n}
$$

Although OWA operator and the weighted mean are both a linear combination of the values, there is a meaningful difference between them due to assigning weight vector. In weighted mean, the $\mathrm{i}$-th data is specified to the i-th weight but in OWA operator weight is specified to the position not to the value.

\section{The Proposed Model}

In this study, the product of three factors, including Severity, Probability, and Detection are considered to calculate RPN as the proposed model by Jamshidi and Wang $[7,8]$ is given as:

$R P N=$ Severity $\times$ Detection $\times$ Probability

Where detection (D) is the probability of a failure detection potential before occurring, probability $(\mathrm{P})$ is the frequency of failure(s) or risk(s) occurrence for a defined device, and severity $(\mathrm{S})$ is the impact of failure on patient's safety, maintenance resources and mean time to repair.

Despite of simplicity of approach, results have some deficiency as following:

- Difference between experts' opinions is ignored.

- Different collections of P, S and D can generate a similar RPN.

- The relative importance between $\mathrm{P}, \mathrm{S}$ and $\mathrm{D}$ is ignored.

In order to overcome these deficits, we applied a modified version of RPN calculation, with considering different experts' opinions and aggregating them using OWA operator. Besides, some linguistic variables are assigned to RPN factors. All of the criteria and subdivision are defined in follow. Table 1 represents an evaluation sheet. Now, we have a multi expert-multi criteria decision making (MEMCDM) problem. The ranking system following comes with a two-stage system. In the first step, experts are asked to provide evaluation sheet lonely as shown in Table (1). This evaluation consists of a rating on each of the criteria and the ratings are chosen from the scale $\{1,2$, $3,4,5\}$, where 5, 4, 3, 2 and 1 stand for very high, high, moderate, low and rare, respectively The next step is to find a general evaluation by a given expert for an alternative. Finally we need to fuse the opinions of experts.

Detectability (D)

This factor refers to the probability of failure 
Table 1: Evaluation sheet

\begin{tabular}{cccccc}
$\begin{array}{c}\text { Risk Priority } \\
\text { Index }\end{array}$ & $\begin{array}{c}\text { Very } \\
\text { High }\end{array}$ & High & Medium & Low & Rare \\
\hline Detectability & & & & & \\
\hline $\begin{array}{c}\text { Probability of } \\
\text { non-detection } \\
\left(D_{1}\right)\end{array}$ & $\bigcirc$ & $\bigcirc$ & $\bigcirc$ & $\bigcirc$ & $\bigcirc$ \\
\hline $\begin{array}{c}\text { Method of } \\
\text { failure detection } \\
\left(D_{2}\right)\end{array}$ & $\bigcirc$ & $\bigcirc$ & $\bigcirc$ & $\bigcirc$ & $\bigcirc$ \\
\hline
\end{tabular}

\section{Probability}

\begin{tabular}{|c|c|c|c|c|c|}
\hline $\begin{array}{c}\text { Mean time } \\
\text { between failures } \\
\left(\mathrm{P}_{1}\right)\end{array}$ & 0 & $O$ & 0 & 0 & $C$ \\
\hline $\begin{array}{c}\text { Repeatability } \\
\left(\mathrm{P}_{2}\right)\end{array}$ & $O$ & $O$ & $O$ & 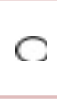 & $C$ \\
\hline
\end{tabular}

\begin{tabular}{|c|c|c|c|c|c|}
\hline Visibility $\left(P_{3}\right)$ & 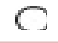 & C & o & $O$ & 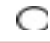 \\
\hline \multicolumn{6}{|l|}{ Failure's } \\
\hline Severity & & & & & \\
\hline $\begin{array}{l}\text { Patient safety } \\
\left(\mathrm{S}_{1}\right)\end{array}$ & $O$ & $\sigma$ & $O$ & 0 & C \\
\hline $\begin{array}{c}\text { Potential risk } \\
\text { for operator and } \\
\text { maintenance } \\
\text { staff }\left(\mathrm{S}_{2}\right)\end{array}$ & 0 & 0 & $O$ & 0 & 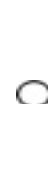 \\
\hline $\begin{array}{l}\text { Mean time to } \\
\text { repair }\left(\mathrm{S}_{3}\right)\end{array}$ & 0 & 0 & 0 & 0 & $\odot$ \\
\hline
\end{tabular}

detection before occurring. In this study, detectability includes two following subdivision as follows:

- Probability of detection $\left(\mathrm{D}_{1}\right)$

- Failure detection method $\left(\mathrm{D}_{2}\right)$

Fuzzy rating and leveling of detectability criteria are demonstrated in Table (2).

\section{Probability}

This factor refers to the frequency of potential failure (s) or risk (s) occurrence probability for an obtained device. In this study, probability includes three following sub-criteria as following:

- Mean time between failures $\left(\mathrm{P}_{1}\right)$

- Repeatability $\left(\mathrm{P}_{2}\right)$

- Visibility $\left(\mathrm{P}_{3}\right)$

Fuzzy rating and leveling of probability criteria are demonstrated in Table (3).

\section{Failure's severity (S)}

This factor refers to the impact of failure severity and probability of risk on the patient and maintenance personnel's safety. In this study, failure's severity includes three following sub-criteria.

- Patient safety $\left(\mathrm{S}_{1}\right)$

- Potential risk for operator and maintenance staff $\left(\mathrm{S}_{2}\right)$

- Mean time to repair $\left(\mathrm{S}_{3}\right)$

Fuzzy rating and leveling of failure's severity criteria are demonstrated in Table (4).

Therefore, we have a multi expert-multi cri-

Table 2: Fuzzy rating and leveling of detectability sub-criteria.

\begin{tabular}{ccc} 
Level & $\mathbf{D}_{\mathbf{1}}$ & $\mathbf{D}_{\mathbf{2}}$ \\
\hline Rare & No scheduled inspection & $\begin{array}{c}\text { No inspection process, the device failures even with a } \\
\text { complete inspection can hardly detected }\end{array}$ \\
\hline Low & Scheduled inspection & $\begin{array}{c}\text { No inspection process, the failure has been allowed to } \\
\text { occur }\end{array}$ \\
\hline Moderate & Detection after an inspection & Manual inspection for some component \\
\hline High & Detection via automatic diagnostic aids & Complete manual inspection \\
\hline Very High & Full visibility detection assessment & $100 \%$ automatic inspection process
\end{tabular}


Table 3: Fuzzy rating and leveling of Probability sub-criteria.

\begin{tabular}{cccc} 
Level & $\mathbf{P}_{\mathbf{1}}$ & $\mathbf{P}_{\mathbf{2}}$ & $\mathbf{P}_{3}$ \\
\hline Very High & Failure is almost inevitable (3 month) & Same failure in 3 months & It is not visible at all \\
\hline High & Repeated failure (3-6 month) & Same failure in 3-6 months & $\begin{array}{c}\text { Visible while using the } \\
\text { device }\end{array}$ \\
\hline Moderate & $\begin{array}{c}\text { Occasional failure (6 month to 24 } \\
\text { months) }\end{array}$ & Same failure in 6-24 months & $\begin{array}{c}\text { Visible between two inspec- } \\
\text { tion intervals }\end{array}$ \\
\hline Low & Relatively few failure (2-5 year) & Same failure in 2-5 years & Visible while inspecting \\
\hline Rare & Failure is ulikely (5 year) & Failure is ulikely (5 year) & Visible before an inspection
\end{tabular}

Table 4: Fuzzy rating and leveling of failure's severity occurrence sub-criteria.

\begin{tabular}{ccc} 
Level & $\mathbf{S}_{\mathbf{1}}$ \& $\mathbf{S}_{\mathbf{2}}$ & $\mathbf{S}_{\mathbf{3}}$ \\
\hline Very High & Death & $\begin{array}{c}\text { Order a new } \\
\text { device }\end{array}$ \\
\hline High & $\begin{array}{c}\text { Debilitating long- } \\
\text { term injury }\end{array}$ & $\begin{array}{c}\text { External interven- } \\
\text { tion for repairs }\end{array}$ \\
\hline Moderate & Moderate injury & $\begin{array}{c}\text { 1 day MTTR 4 } \\
\text { days }\end{array}$ \\
\hline Low & Minor injury & 1 h MTTR 1 day \\
\hline Rare & Less no effect & MTTR 1 h
\end{tabular}

MTTR: Mean time to return teria decision making (ME-MCDM) problem. The ranking system is a two-step process as explained in the following. In the first step, individual experts are asked to provide an evaluation of the selected character. This assessment includes a ratio for each variable at any scale, and the ratios for each scale are $\{1,2$, 3 \} that 3,2 and 1 show excellent, average and weak performance, respectively. Each expert provides a 6-tuple $\left(a_{1}, a_{2}, a_{3}, a_{4}, a_{5}, a_{6}\right)$ for each applicant, where $a_{i} \in\{1,2,3\}, i=1,2,3,4,5$, 6 . The next step is finding the overall evaluation for each expert on selected character. Aggregation of experts' opinion for different medical devices on all sub-criteria is shown in Table (5).

Table 5: Aggregation of experts' opinion for all sub-criteria.

\begin{tabular}{|c|c|c|c|c|c|c|c|c|c|}
\hline \multirow{3}{*}{ Medical Device } & \multicolumn{9}{|c|}{ Risk Priority Index } \\
\hline & \multicolumn{3}{|c|}{ Detectability } & \multicolumn{3}{|c|}{ Probability } & \multicolumn{3}{|c|}{ Severity } \\
\hline & $\mathbf{L}$ & $\mathbf{M}$ & $\mathbf{U}$ & $\mathbf{L}$ & $\mathbf{M}$ & $\mathbf{U}$ & $\mathbf{L}$ & $\mathbf{M}$ & $\mathbf{U}$ \\
\hline$X X$ & & & & & & & & & \\
\hline
\end{tabular}

Lower=L, Medium=M, Upper=U 
Results

This section provides a numerical example of proposed model. Table (6) shows multiple experts' opinion on all sub-criteria for four chosen medical devices; Defibrillator, Infusion Pump, Surgical Light and Surgical Suction; based on the evaluation sheet is drawn in Table (1). The ratings and leveling indexes are

Table 6: Opinion of expert

Fuzzy Risk Priority Level

\begin{tabular}{|c|c|c|c|c|c|c|c|c|c|}
\hline \multirow{3}{*}{ Medical Device } & \multirow{3}{*}{ Expert } & \multirow{2}{*}{\multicolumn{2}{|c|}{ Detectability }} & \multirow{2}{*}{\multicolumn{3}{|c|}{ Probability }} & \multirow{2}{*}{\multicolumn{3}{|c|}{ Severity }} \\
\hline & & & & & & & & & \\
\hline & & $D_{1}$ & $\mathrm{D}_{2}$ & $P_{1}$ & $\mathbf{P}_{2}$ & $\mathbf{P}_{3}$ & $S_{1}$ & $S_{2}$ & $S_{3}$ \\
\hline \multirow{5}{*}{ Defibrillator } & Expert 1 & 5 & 5 & 4 & 4 & 4 & 4 & 5 & 4 \\
\hline & Expert 2 & 4 & 4 & 3 & 2 & 2 & 3 & 4 & 4 \\
\hline & Expert 3 & 4 & 5 & 4 & 3 & 4 & 4 & 4 & 3 \\
\hline & Expert 4 & 5 & 5 & 3 & 3 & 3 & 4 & 5 & 3 \\
\hline & Expert 5 & 4 & 5 & 3 & 3 & 4 & 3 & 5 & 4 \\
\hline \multirow{5}{*}{ Infusion Pump } & Expert 1 & 4 & 4 & 3 & 3 & 4 & 4 & 3 & 3 \\
\hline & Expert 2 & 4 & 3 & 3 & 3 & 3 & 4 & 3 & 3 \\
\hline & Expert 3 & 3 & 3 & 3 & 2 & 2 & 3 & 3 & 3 \\
\hline & Expert 4 & 4 & 5 & 3 & 2 & 3 & 3 & 3 & 2 \\
\hline & Expert 5 & 4 & 3 & 4 & 2 & 3 & 4 & 4 & 3 \\
\hline \multirow{5}{*}{ Surgical Light } & Expert 1 & 3 & 3 & 3 & 3 & 2 & 3 & 4 & 4 \\
\hline & Expert 2 & 3 & 3 & 2 & 3 & 2 & 2 & 3 & 3 \\
\hline & Expert 3 & 2 & 3 & 3 & 2 & 2 & 2 & 3 & 3 \\
\hline & Expert 4 & 3 & 3 & 2 & 2 & 2 & 2 & 3 & 3 \\
\hline & Expert 5 & 3 & 3 & 2 & 2 & 3 & 3 & 3 & 3 \\
\hline \multirow{6}{*}{ Surgical Suction } & Expert 1 & 3 & 3 & 2 & 3 & 2 & 3 & 4 & 3 \\
\hline & Expert 2 & 4 & 4 & 3 & 3 & 3 & 3 & 3 & 2 \\
\hline & Expert 3 & 3 & 3 & 3 & 2 & 2 & 3 & 2 & 2 \\
\hline & Expert 4 & 3 & 4 & 3 & 2 & 2 & 3 & 2 & 2 \\
\hline & Expert 5 & 4 & 3 & 3 & 2 & 3 & 4 & 3 & 3 \\
\hline & Low=2 & \multicolumn{2}{|c|}{ Moderate $=3$} & \multicolumn{2}{|c|}{ High $=4$} & \multicolumn{4}{|c|}{ Very High=5 } \\
\hline
\end{tabular}


chosen from the scale $\{5,4,3,2,1\}$, where 5 , 4, 3, 2 and 1 are for very high, high, moderate, low and rare, respectively. Each expert scores sub-criteria $\left(\mathrm{D}_{1}, \mathrm{D}_{2}, \mathrm{O}_{1}, \mathrm{O}_{2}, \mathrm{O}_{3}, \mathrm{~S}_{1}, \mathrm{~S}_{2} \mathrm{~S}_{3}\right)$ for each medical device. Considering an Orness value reflect optimistic value, we calculate the RPN value based on each expert's point of view. Considering 0.9 for orness, we find the value of RPN, which is shown in Table (6).

The next step is finding the overall evaluation for an alternative based on OWA operator derived from an appropriate linguistic quantifier. The results of expert opinion fusion for four selected medical devices are shown in Table (7).

\section{Discussion}

Calculation of RPN is vital in maintenance program of medical device. Limited budget is more important in medical centers. In this study, we have introduced the application of OWA operator for aggregation of different parameters for calculating of RPN. This framework presents a simple, precise and reliable method to choose the best maintenance policy for each medical device based on its importance. Based on the obtained results from numerical model, defibrillator and surgical suction have the highest and the lowest ranking between other chosen medical devices as shown in Table 3.

\section{Conclusion}

The study aims to propose and develop a risk assessment method and maintenance strategy for medical devices of medical centers and hospitals. For healthcare organizations, pri- oritization of medical devices based on risk is valuable because our future work is the development of a risk-based maintenance software based on this framework for implementing in healthcare center.

\section{Conflict of Interest}

None

\section{References}

1. Dyro JF. Clinical Engineering Handbook. Amsterdam: Elsevier; 2003.

2. Fennigkoh L, Smith B. Clinical equipment management. JCAHO PTSM Series. 1989;2:5-14.

3. Joint Commission on Accreditation of Healthcare Organizations. Hospital accreditation standards, Oakbrook Terrace, IL. JACAHO; 2004.

4. Tawfik B, Ouda BK, El Samad YMA. A fuzzy logic model for medical equipment risk classification. J Clin Eng. 2013;38:185-90. doi: 10.1097/jce.0b013e3182a90445.

5. American Society for Healthcare Engineering of the American Hospital Association. Clinical Equipment Risk Classification. USA: ASHE/ AHA; 1996.

6. Yager RR. On ordered weighted averaging aggregation operators in multicriteria decisionmaking. IEEE Transactions on Systems, Man, and Cybernetics. 1988;18:183-90. doi: 10.1109/21.87068.

7. Jamshidi A, Rahimi SA, Ait-Kadi D, Ruiz A. A comprehensive fuzzy risk-based maintenance framework for prioritization of medical devices. Applied Soft Computing. 2015;32:32234. doi: 10.1016/j.asoc.2015.03.054.

Table 7: Final Risk Priority Number (RPN) based on fusion of expert opinion.

Medical Device Defibrillator Infusion Pump Surgical Light Surgical Suction

$\begin{array}{lllll}\text { RPN } & 4.04 & 3.99 & 3.21 & 3.08\end{array}$

RPN: Risk Priority Number 
Fereshteh Azadi Parand, et al

8. Wang Y-M, Chin K-S, Poon GKK, Yang

metric mean. Expert Systems with Applica$\mathrm{J}$-B. Risk evaluation in failure mode and effects analysis using fuzzy weighted geo-

tions. 2009;36:1195-207. doi: 10.1016/j. eswa.2007.11.028. 